\title{
Application of Two-Dimensional Correlation UV-Vis Spectroscopy in Chinese Liquor Moutai Discrimination
}

\author{
Zhengyong Zhang1,2, Jiuying Jiang1,2, Guoxiang Wang1,2, Hu Wang1,2, Jun Liu, ${ }^{1,2}$, \\ Haiyan Wang1,2* \\ ${ }^{1}$ School of Management Science \& Engineering, Nanjing University of Finance \& Economics, Nanjing, China \\ 2Jiangsu Province Institute of Quality \& Safety Engineering, Nanjing, China \\ Email: ${ }^{*}$ njue 10@163.com
}

Received 21 February 2015; accepted 27 March 2015; published 31 March 2015

Copyright (C 2015 by authors and Scientific Research Publishing Inc.

This work is licensed under the Creative Commons Attribution International License (CC BY).

http://creativecommons.org/licenses/by/4.0/

c) (i) Open Access

\begin{abstract}
Chinese liquor Moutai is the "National alcoholic drink" in China and plays a very important role of social activities in Chinese people's life. In pursuit of high profits, some illegal counterfeit Moutai liquors have begun to appear in the market. Therefore, it is an urgent need for new techniques to discriminate the genuine and counterfeit Moutai liquor. In this work, the conventional Ultraviolet-Visible (UV-Vis) spectroscopy and two-dimensional correlation UV-Vis spectroscopy are applied to obtain the UV-Vis characteristic of Moutai liquor and counterfeit one, respectively. The experimental results reveal that the conventional UV-Vis spectra of the genuine and counterfeit Moutai liquor are similar. However, the two-dimensional correlation UV-Vis spectra of them are different and this method would be applied to differentiate the counterfeit Moutai liquor from the genuine Moutai liquor. Compared with conventional methods, this novel method has the advantages of easy operation, simple instrumentation and direct recognition, which make it a potential tool in the fields of food safety.
\end{abstract}

\section{Keywords}

Two-Dimensional Correlation Spectra, UV-Vis Spectroscopy, Moutai Liquor, Discrimination

\section{Introduction}

Chinese liquor is one of the oldest distilled spirits in the world and plays a significant role in Chinese people's

\footnotetext{
*Corresponding author.
}

How to cite this paper: Zhang, Z.Y., Jiang, J.Y., Wang, G.X., Wang, H., Liu, J. and Wang, H.Y. (2015) Application of Two-Dimensional Correlation UV-Vis Spectroscopy in Chinese Liquor Moutai Discrimination. American Journal of Analytical Chemistry, 6, 395-401. http://dx.doi.org/10.4236/ajac.2015.65038 
life [1]. The Chinese liquor Moutai (or Maotai) is the most well-known Chinese liquor, owing to its long history of distillation, excellent and unique qualities and complex production processes [2]. It is usually fermented from grains, mainly involving sorghum and wheat, and after several months or years of fermentation, the liquor is distilled out with steam. Then the fresh distillates are aged for several months or years in order to balance the different aromatic compounds, and finally, the aged liquors are diluted with water and blended into the products [3]-[5]. Additionally, based on the unique geographical environment of Moutai town in China, the Moutai liquor owns the unusual fragrance [6], and it has ever won the gold medal at Panama Pan-world Fair in 1915 [7]. Right now it has been dubbed the title of the "National alcoholic drink" in China and has great demands in more than one hundred countries in the world [8] [9]. Under the tremendous economic temptation, some illegal traders start using counterfeit Moutai liquors to cheat the customers. Hence, it is highly required to authenticate the Moutai liquor [10]. However, the most common detection strategy for analysis of Chinese liquors is integrity by component approach, such as gas chromatography, mass spectrometry and gas chromatograph-mass spectrometry [11]-[14]. These techniques enable identification and quantification of hundreds of compounds of Chinese liquors, but the experimental processes are time-consuming and the instruments are expensive and large-scale, which limit their practical applications [15]-[17]. Therefore, the quality management of Chinese liquors still mainly depends on a traditional technique, by hiring the oenophiles to taste the aroma and flavor of Chinese liquors and identify their differences [18]. But the accuracy and objectivity cannot be always guaranteed because oenophiles may be affected by their own healthy status, feeling and external environment. Especially, counterfeit liquors, which may be produced by mixing industrial alcohol with water, are not suitable for tasting and discriminating by oenophiles [19].

In recent years, the rapid development has been gained in the applications of spectroscopic techniques in Chinese liquors discrimination. Particularly, the Infrared (IR) spectroscopy is usually employed to identify the Chinese liquors owing to its simple pretreatment for a sample [20]. However, the water in the liquor also has an IR adsorption peak which may interfere with the IR results of the sample. Ultraviolet-Visible (UV-Vis) spectroscopy and two-dimensional correlation spectroscopy could be used as another kind of tool for identification and discrimination of Chinese liquors, and up to now, there have been still few studies on this. The two-dimensional correlation spectroscopy method was developed by Noda et al. [21]-[23], and it has become a very useful analytical technique for complex mixture systems, because the technique is based on a series of perturbation-induced dynamic spectra and a simple scheme of correlation analysis, then generates clearly and easily identifiable graphical representation of spectral fingerprints [24]-[26]. Herein, we evaluate and identify the genuine Moutai liquor and counterfeit Moutai liquor by using the two-dimensional correlation UV-Vis spectroscopy technique.

\section{Experimental}

\subsection{Reagents and Instruments}

Absolute ethanol and sodium hydroxide were purchased from Xilong Chemical Co., Ltd. (Shantou, China). Moutai liquor was obtained from Kweichow Moutai Co., Ltd. (Guizhou, China). Counterfeit Moutai liquor was obtained from China National Research Institute of Food and Fermentation Industries. Double distilled water was used throughout.

The UV-Visible absorption data were recorded from a UV-Vis spectrometer, T6 New century, Purkinje General Instrument Co., Ltd. (Beijing, China).

\subsection{Methods}

\subsubsection{Sodium Hydroxide Concentration Optimization}

$2 \mathrm{~mL}$ liquor sample was added into a glass of bottle, then different concentrations of $400 \mu \mathrm{L}$ sodium hydroxide solution $(0.2625 \mathrm{~mol} / \mathrm{L}, 0.4375 \mathrm{~mol} / \mathrm{L}, 0.875 \mathrm{~mol} / \mathrm{L}, 1.3125 \mathrm{~mol} / \mathrm{L}, 1.75 \mathrm{~mol} / \mathrm{L}, 2.1875 \mathrm{~mol} / \mathrm{L}, 2.625 \mathrm{~mol} / \mathrm{L}$, $3.0625 \mathrm{~mol} / \mathrm{L}, 3.5 \mathrm{~mol} / \mathrm{L}$ ) was added to each bottle, respectively. Next, the mixture solution was left to react in an oven at $70^{\circ} \mathrm{C}$ for $4 \mathrm{~h}$. At last, the UV-Vis spectrum of the reaction solution was collected by a UV-Vis spectrometer, respectively.

\subsubsection{UV-Vis Spectroscopy}

$2 \mathrm{~mL}$ liquor sample was added into a glass of bottle, then $400 \mu \mathrm{L}$ sodium hydroxide solution $(1.75 \mathrm{~mol} / \mathrm{L})$ was 
added to the liquor, and the mixture solution was left to react in an oven at $70^{\circ} \mathrm{C}$, Next, the UV-Vis spectrum of the reaction solution was collected once by a UV-Vis spectrometer every $30 \min$ for $4 \mathrm{~h}$ or $5 \mathrm{~min}$ for $1 \mathrm{~h}$. Those collected spectra were used as the dynamic spectra in the next two-dimensional correlation analysis.

\subsubsection{Two-Dimensional UV-Vis Correlation Spectroscopy}

The graphs of two-dimensional correlation UV-Vis spectra are obtained by the treatment of the series of UV-Vis dynamic spectra with the software "2D-shige", which is being developed by Shigeaki Morita in Professor Yukihiro Ozaki’s group at Kwansei-Gakuin University. In the two-dimensional correlation analysis, the average spectrum is used as the reference spectrum, the positive and negative correlation intensities are denoted by the unshaded regions and shaded regions [27].

\section{Results and Discussion}

The sample of Chinese liquor Moutai is a complex mixture system, which usually contains hundreds of fragrant and flavor compounds [28]. Further studies reveal that the complexity of Chinese liquor Moutai in the chemical classes is mainly including alcohols, esters, acids, aldehydes, ketones, phenols and nitrogen-heterocyclic and so on [29] [30]. Hence, it is difficult to well identify the quality of Moutai liquor by using the current commercial separation and analysis techniques. In contrast, the spectrum detection technique is expected to provide a new testing strategy [31]. Figure 1 shows the UV-Vis spectra of the Moutai liquor and counterfeit Moutai liquor. By comparing their UV-Vis spectra, the similar absorption peaks of them at $278 \mathrm{~nm}$ were shown in the results, which were mainly attributed to absorption of the furfural molecule [32]. The difference of them was only reflected on the absorption intensity, thus it is uneasy to get the extract useful information for directly identification of the samples from the conventional UV-Vis results.

Recently, the two-dimensional correlation spectroscopy technique has been developed because it can improve the resolution of a spectrum through the difference in responses of various molecular groups of a system to a given external perturbation [33]-[35]. Besides, it can provide some new information, which cannot be obtained from the conventional spectrum. Therefore, there is a huge advantage by using two-dimensional correlation spectroscopy to identify and discriminate the complex system [36]. In this paper, two-dimensional correlation UV-Vis spectra were obtained based on the sodium hydroxide induced chemical reaction. Firstly, the UV-Vis spectra of sample's mixture solution during the reaction process were measured as the dynamic spectra. Subsequently, the collected spectra were converted into a two-dimensional correlation UV-Vis spectrum. It has been reported that the Moutai liquor could occur color reaction at $70^{\circ} \mathrm{C}$ for $4 \mathrm{~h}$ under the alkaline solutions [37]. As shown in the Figure 2, different concentration of sodium hydroxide solutions were mixed with Moutai liquor, respectively, and the corresponding reaction results were measured by a UV-Vis spectrometer. The results display that the UV-Vis adsorption spectra is red-shift with the increase of sodium hydroxide solution, and when the amount of the added sodium hydroxide solution reached $1.75 \mathrm{~mol} / \mathrm{L}$, the reaction tends to be stable.

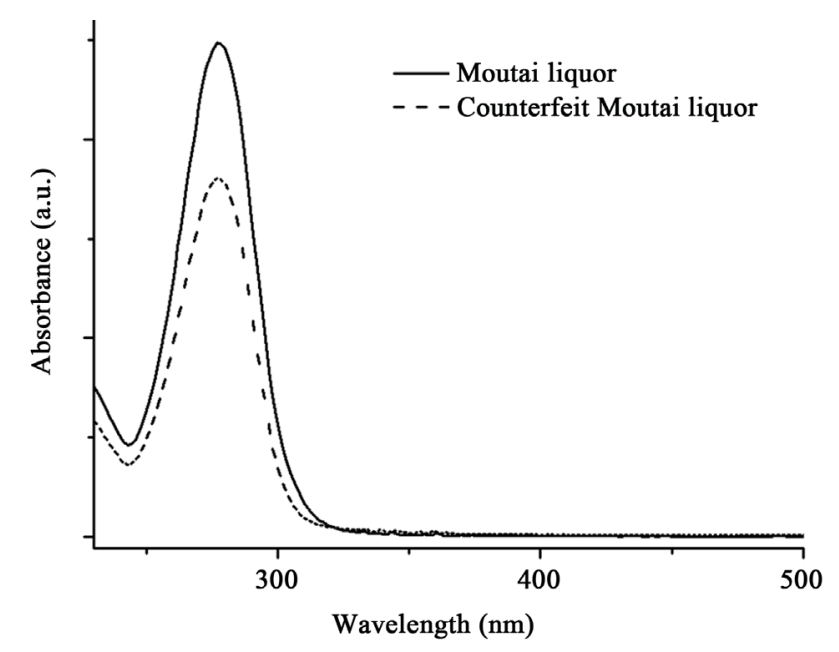

Figure 1. UV-Vis spectra of Moutai liquor (solid line) and counterfeit Moutai liquor (dash line). 


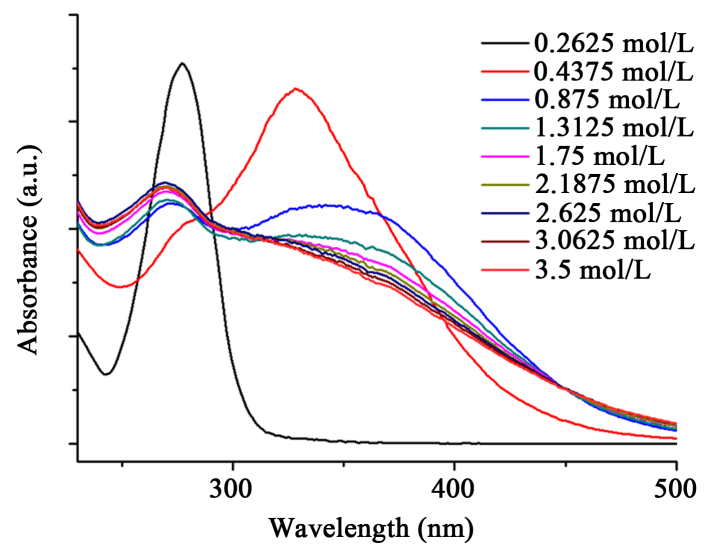

Figure 2. UV-Vis spectra of Moutai liquor with different concentration sodium hydroxide solutions.

Figure 3 shows the dynamic UV-Vis spectra and two-dimensional correlation synchronous spectra of Moutai liquor and counterfeit Mourtai liquor in the wavelength range of $250-500 \mathrm{~nm}$. As shown in the conventional UV-Vis spectra (Figure 3(a) and Figure 3(c)), which are collected of the react system under the alkaline solutions every $30 \mathrm{~min}$ for $4 \mathrm{~h}$, it can be found that the absorption peaks of both of Moutai liquor and counterfeit Moutai liquor have a similar shift trend, but the changed peaks are around $350 \mathrm{~nm}$ and $330 \mathrm{~nm}$, respectively. The spectra change of the reaction (color change of Moutai liquor under the alkaline condition) was mainly induced by the organic compounds including furfural, diacetyl, acetoin, and 2,3-diketone, etc. It has been reported that most of these compounds contained carbonyl or conjugate $\pi$ bond, after heating under alkaline condition, intramolecular electron transfer and rearrangement of them would form cross conjugated system and further prolong $\pi$ bond. Plus, heterocyclic compound in the liquor such as furan and pyran would form multiple electron conjugated system for multi $\pi$ aromatic heterocycles, so that the reaction results display apparent spectra changes finally [38]-[40].

From the two-dimensional correlation UV-Vis spectra, the minimal spectra changes (color change of Moutai liquor under the alkaline condition) have been expanded, which is directly shown in the Figure 3(b) and Figure $3(d)$, respectively. In the two-dimensional correlation graphs unshaded regions indicate positive correlation signs, while shaded regions indicate negative correlation signs. The Moutai liquor has one strong auto-peak at $345 \mathrm{~nm}$ and another auto-peak at $278 \mathrm{~nm}$ with positive intensities, and a pair of cross peaks at ( $278 \mathrm{~nm}, 345 \mathrm{~nm}$ ) and (345 $\mathrm{nm}, 278 \mathrm{~nm}$ ) with negative intensities. For the counterfeit Moutai liquor, it has one strong auto-peak at $328 \mathrm{~nm}$ and an auto-peak at $278 \mathrm{~nm}$ with positive intensities, and a pair of cross-peak at $(278 \mathrm{~nm}, 328 \mathrm{~nm})$ and $(328 \mathrm{~nm}, 278$ $\mathrm{nm}$ ) with negative intensities. Additionally, the positive range of them is in $300-460 \mathrm{~nm}$ and $300-410 \mathrm{~nm}$, respectively. Hence, by using such different two-dimensional correlation UV-Vis spectra, a new interesting tool for the discrimination of counterfeit Moutai liquor from genuine Moutai liquor was developed.

Furthermore, the dynamic UV-Vis spectra in the range of 0 - $30 \mathrm{~min}$ and 0 - $1 \mathrm{~h}$ were evaluated in this work, respectively, as shown in Figure 4. For the conventional UV-Vis spectra (Figure 4(a) and Figure 4(d)), the absorption spectra of Moutai liquor were changed rapider than the counterfeit Moutai liquor at the same condition, and the red-shift trend was similar as above mentioned in the Figure 3(a) and Figure 3(c), but their two-dimensional correlation spectra are significantly different. Specifically, Figure 4(b) and Figure 4(e) $(\mathrm{t}=0$ - $30 \mathrm{~min})$ have the similar differences as the above mentioned in Figure 3(b) and Figure 3(d). However, the Figure 4(c) and Figure 4(f) $(\mathrm{t}=0-1 \mathrm{~h})$ display new differences. Moutai liquor has a unique auto-peak around $345 \mathrm{~nm}$ and another auto-peak at $277 \mathrm{~nm}$ with positive intensities in the Figure 4(c), the cross peaks of Moutai liquor were situated at $(277 \mathrm{~nm}, 333 \mathrm{~nm})$ and $(333 \mathrm{~nm}, 277 \mathrm{~nm})$ with negative intensities, respectively. For counterfeit Moutai liquor (Figure 4(f)), there are two obvious auto-peaks at $277 \mathrm{~nm}$ and $328 \mathrm{~nm}$ with positive intensities, and a pair of cross peaks at $(277 \mathrm{~nm}, 328 \mathrm{~nm})$ and $(328 \mathrm{~nm}, 277 \mathrm{~nm})$ with negative intensities. The peak positions in the twodimensional correlation UV-Vis spectra of counterfeit Moutai liquor are different from genuine Moutai liquor. In addition, 0 - $1 \mathrm{~h}$ could be chose as the optimum reaction time in this work. These characteristics of the two-dimensional correlation UV-Vis spectra are visually so evident, so that this method has the potential in directly used for the quality analysis of Moutai liquor. 

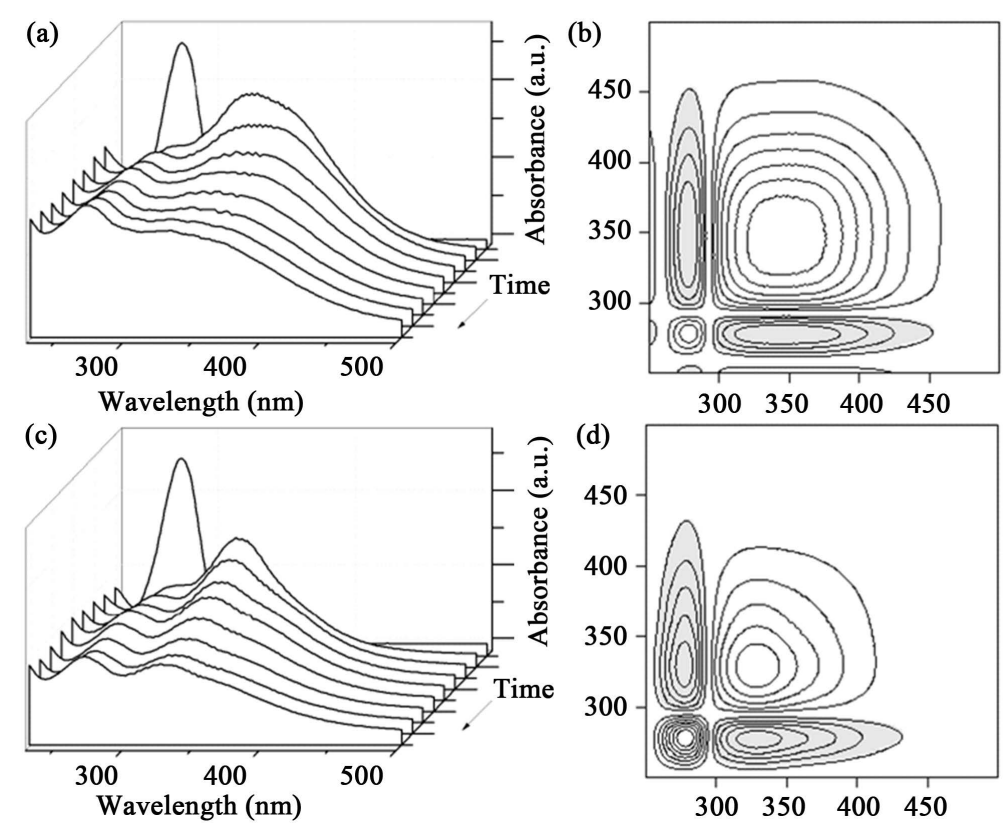

Figure 3. UV-Vis spectra of Moutai liquor $((\mathrm{a}), \mathrm{t}=0-4 \mathrm{~h}, \Delta \mathrm{t}=0.5 \mathrm{~h})$ and counterfeit Moutai liquor ((c), $\mathrm{t}=0-4 \mathrm{~h}, \Delta \mathrm{t}=0.5 \mathrm{~h})$, two-dimensional correlation UV-Vis spectra of Moutai liquor (b) and counterfeit Moutai liquor (d) based on the previous dynamic UV-Vis spectra, respectively.
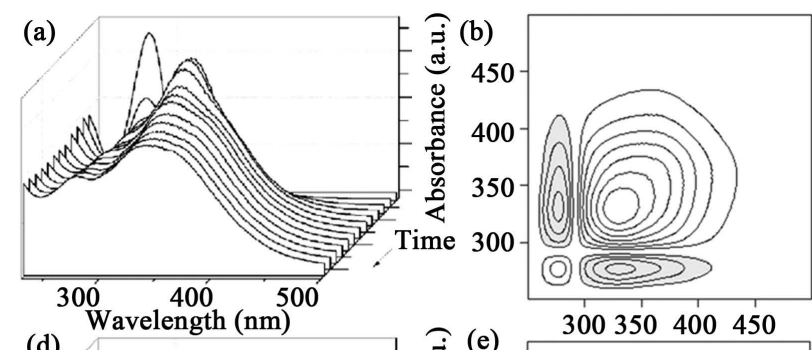

(d)
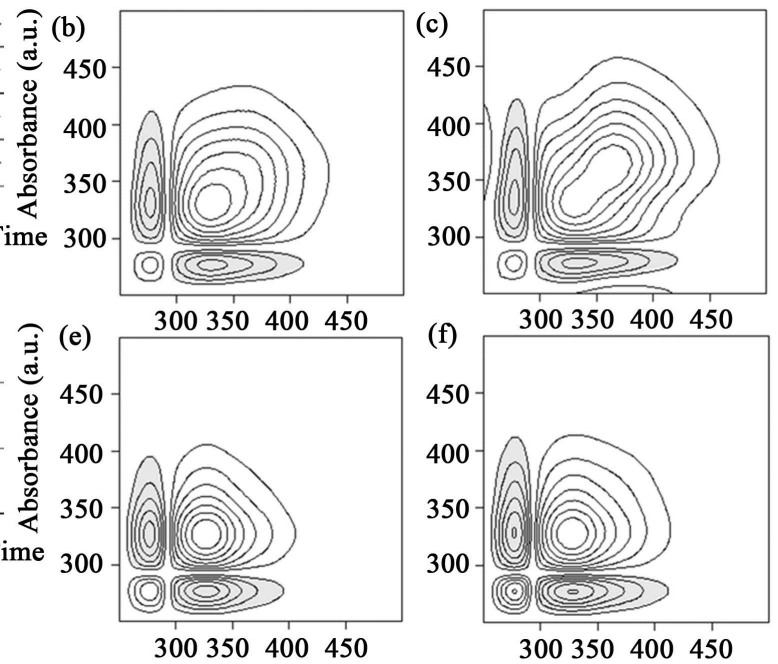

Figure 4. UV-Vis spectra of Moutai liquor ((a), $t=0-1 \mathrm{~h}, \Delta \mathrm{t}=5 \mathrm{~min})$ and counterfeit Moutai liquor ((d), $\mathrm{t}=0-1 \mathrm{~h}, \Delta \mathrm{t}=5 \mathrm{~min})$, two-dimensional correlation UV-Vis spectra of Moutai liquor ((b), $\mathrm{t}=0-30 \mathrm{~min}$ and (c), $\mathrm{t}=0-1 \mathrm{~h}$ ) and counterfeit Moutai liquor ((e), $\mathrm{t}=0-30 \mathrm{~min}$ and (f), $\mathrm{t}=0-1 \mathrm{~h}$ ) based on the previous dynamic UV-Vis spectra, respectively.

\section{Conclusion}

In summary, the conventional UV-Vis spectroscopy and two-dimensional correlation UV-Vis spectroscopy are applied to the identification and discrimination of the authentic and adulterated samples of Chinese liquor Moutai in this work, respectively. The obtained results suggested that the novel two-dimensional correlation UV-Vis spectroscopy analytical method has the potential application in the identification and discrimination of the counterfeit Moutai liquor from genuine Moutai liquor. The potential applications of this strategy also could be most likely extended to the evaluation of other fields. 


\section{Acknowledgements}

We gratefully acknowledge the financial support of this research by National Natural Science Foundation of China $(61373058,71433006)$ and the scientific research foundation of General Administration of Quality Supervision, Inspection and Quarantine of China (2012104009-2, 201410173), and the Scientific Research Foundation for Advanced Talent of Nanjing University of Finance \& Economics.

\section{References}

[1] Zheng, Y., Tang, Q., Wu, T., Tian, Z., Zhang, X. and Zhang, Q. (2008) Study on Fingerprint Construction of Kweichow Moutailiqour by GC-MS. China Brewing, 9, 74-91.

[2] Fan, G., Wang, H., Cui, T., Chen, A., Jiang, H., Jiang, P., Wang, L. and Guo, K. (2006) Researching Development of Maotai Microorganisms. Liquor-Making Science \& Technology, 10, 75-77.

[3] National Standards of the People’s Republic of China (2007) Product of Geographical Indication-Kweichow Moutai Liquor.

[4] Hu, P., Qiu, S., Wu, X., Chen, L. and Li, J. (2009) The Stabilization of Maotai-flavor Liquor Production Techniques Is the Base for the Development of Grade II \& Grade III Maotai-Flavor Liqour-Making Enterpries. Liquor-Making Science \& Technology, 10, 110-113.

[5] Shen, Y. (2009) The Whole Book of Chinese Liquor Production Techniques. China Light Industry Press, Beijing.

[6] Cheng, P., Fan, W. and Xu, Y. (2014) Determination of Chinese Liquors from Different Geographic Origins by Combination of Mass Spectrometry and Chemometric Technique. Food Control, 35, 153-158. http://dx.doi.org/10.1016/j.foodcont.2013.07.003

[7] Zhu, S., Lu, X., Ji, K., Guo, K., Li, Y., Wu, C. and Xu, G. (2007) Characterization of Flavor Compounds in Chinese Liquor Moutai by Comprehensive Two-Dimensional Gas Chromatography/Time-of-Flight Mass Spectrometry. Analytica Chimica Acta, 597, 340-348. http://dx.doi.org/10.1016/j.aca.2007.07.007

[8] Ji, K. (2003) The Effects, Historical Situations and Functions of Maotai Liquor in the Development of Liqours in China. Liquor-Making Science \& Technology, 4, 29-31.

[9] Chen, X. and Ji, K. (2006) General Introduction to the Individualities of Maotai Liquor. Liquor-Making Science Technology, 2, 79-84.

[10] Zhen, C., Zhou, Y., Zhang, N., Wang, J., Xiong, C., Chen, S. and Nie, Z. (2013) Differentiation of Chinese Liquors by Using Ambient Glow Discharge Ionization Mass Spectrometry. Analyst, 138, 3830-3835. http://dx.doi.org/10.1039/c3an00074e

[11] Fan, W. and Qian, M.C. (2006) Identification of Aroma Compounds in Chinese 'YangheDaqu' Liquor by Normal Phase Chromatography Fractionation Followed by Gas Chromatography/Olfactometry. Flavour and Fragrance Journal, 21, 333-342.

[12] Du, H. and Xu, Y. (2012) Determination of the Microbial Origin of Geosmin in Chinese Liquor. Journal of Agricultural and Food Chemistry, 60, 2288-2292. http://dx.doi.org/10.1021/jf204648e

[13] National Standards of the People's Republic of China (2007) Method of Analysis for Chinese Spirits.

[14] Zhang, C., Ao, Z., Chui, W., Shen, C., Tao, W. and Zhang, S. (2012) Characterization of the Aroma-Active Compounds in Daqu: A Tradition Chinese Liquor Starter. European Food Research and Technology, 234, 69-76. http://dx.doi.org/10.1007/s00217-011-1616-4

[15] Sun, Q., Xiang, P. and Shen, B. (2010) Identification of Kweichow Moutai Liquor by Gas Chromatography-Mass Spectrometry Fingerprint. Chinese Journal of Chromatography, 28, 833-839.

[16] Xiao, Z., Yu, D., Niu, Y., Chen, F., Song, S., Zhu, J. and Zhu, G. (2014) Characterization of Aroma Compounds of Chinese Famous Liquors by Gas Chromatography-Mass Spectrometry and Flash GC Electronic-Nose. Journal of Chromatography B, 945-946, 92-100. http://dx.doi.org/10.1016/j.jchromb.2013.11.032

[17] Qin, H., Huo, D., Zhang, L., Yang, L., Zhang, S., Yang, M., Shen, C. and Hou, C. (2012) Colorimetric Artificial Nose for Identification of Chinese Liquor with Different Geographic Origins. Food Research International, 45, 45-51. http://dx.doi.org/10.1016/j.foodres.2011.09.008

[18] Li, W. (2007) Flavor and Flavor Styles of Liqour. Liquor Making, 34, 5-7.

[19] Zhang, Q., Xie, C., Zhang, S., Wang, A., Zhu, B., Wang, L. and Yang, Z. (2005) Identification and Pattern Recognition Analysis of Chinese Liquors by Doped Nano ZnO Gas Sensor Array. Sensors and Actuators B: Chemical, 110, 370376. http://dx.doi.org/10.1016/j.snb.2005.02.017

[20] Li, C., Wei, J., Sun, S. and Xu, Q. (2006) Identification of Maotai-Flavor Liquors by FT-IR. Liquor-Making Science \& 
Technology, 11, 56-58.

[21] Noda, I. (1989) Two-Dimensional Infrared Spectroscopy. Journal of the American Chemical Society, 111, 8116-8118. http://dx.doi.org/10.1021/ja00203a008

[22] Noda, I. (1993) Generalized Two-Dimensional Correlation Method Applicable to Infrared, Raman, and Other Types of Spectroscopy. Applied Spectroscopy, 47, 1329-1336. http://dx.doi.org/10.1366/0003702934067694

[23] Morita, S., Shinzawa, H., Noda, I. and Ozaki, Y. (2006) Perturbation-Correlation Moving-Window Two-Dimensional Correlation Spectroscopy. Applied Spectroscopy, 60, 398-406. http://dx.doi.org/10.1366/000370206776593690

[24] Noda, I., Dowrey, A.E., Marcoli, C., Story, G.M. and Ozaki, Y. (2000) Generalized Two-Dimensional Correlation Spectroscopy. Applied Spectroscopy, 54, 236A-248A. http://dx.doi.org/10.1366/0003702001950454

[25] Zhou, X., Xiang, B. and Zhang, M. (2013) Novel Spectral Interval Selection Method Based on Synchronous Two-Dimensional Correlation Spectroscopy. Analytical Letters, 46, 340-348. http://dx.doi.org/10.1080/00032719.2012.713068

[26] Quaroni, L., Zlateva, T. and Normand, E. (2011) Detection of Weak Absorption Changes from Molecular Events in Time-Resolved FT-IR Spectromicroscopy Measurements of Single Functional Cells. Analytical Chemistry, 83, 73717380. http://dx.doi.org/10.1021/ac201318z

[27] Guo, Y., Jin, Y. and Su, Z. (2012) Spectroscopic Study of the Microstructure and Phase Transition of RegioregularPoly(3-dodecylthiophene). Soft Matter, 8, 2907-2914. http://dx.doi.org/10.1039/c2sm07191f

[28] Li, C., Wei, J., Zhou, Q. and Sun, S. (2008) Rapid Identification of Chinese Sauce Liquor from Different Fermentation Positions with FT-IR Spectroscopy. Journal of Molecular Structure, 883-884, 99-102. http://dx.doi.org/10.1016/j.molstruc.2007.12.012

[29] Ji, K. and Guo, K. (2006) Investigation on Microconstituents in Maotai Liquor. Liquor-Making Science \& Technology, 10, 98-100.

[30] Zhang, R., Wu, Q. and Xu, Y. (2013) Aroma Characteristics of Moutai-Flavour Liquor Produced with Bacillus Licheniformis by Solid-State Fermentation. Letters in Applied Microbiology, 57, 11-18. http://dx.doi.org/10.1111/lam.12087

[31] Wang, L., Ji, K. and Xu, Y. (2005) Near Infrared Spectroscopy and Its Application Prospect in Quality Control of Liquor. Liquor Making, 32, 17-19.

[32] Jia, T. (2007) The Elementary Study on the Chinese Famous Liquor by Spectral Methods. Master’s Dissertation, Henan University, Kaifeng.

[33] Sun, S., Li, C., Wei, J., Zhou, Q. and Noda, I. (2006) Discrimination of Chinese Sauce Liquor Using FT-IR and TwoDimensional Correlation IR Spectroscopy. Journal of Molecular Structure, 799, 72-76. http://dx.doi.org/10.1016/j.molstruc.2006.03.024

[34] Noda, I. and Ozaki, Y. (2004) Two-Dimensional Correlation Spectroscopy-Applications in Vibrational and Optical Spectroscopy. Wiley, Chichester. http://dx.doi.org/10.1002/0470012404

[35] Huang, H., Ding, X., Zhu, C., He, Z. and Yu, Y. (2013) 2D Correlation Analysis: Sequential Order Judging. Analytical Chemistry, 85, 2161-2168. http://dx.doi.org/10.1021/ac3027355

[36] Noda, I. (2010) Two-Dimensional Correlation Spectroscopy-Biannual Survey 2007-2009. Journal of Molecular Structure, 974, 3-24. http://dx.doi.org/10.1016/j.molstruc.2010.01.069

[37] Wang, D., Hu, Y., Wang, J. and Zhang, S. (2005) Distinguishment of Liquor by Solid Fermentation and Its Content in Liquor Determination. Liquor-Making Science \& Technology, 2, 79-81.

[38] Zhang, Z. and Xiang, S. (2012) Research on Color Change of Liquor Containing Conjugate $\pi$ Bond Polar Compounds in Conditions of Alkaline Heating (I). Liquor-Making Science \& Technology, 8, 77-81.

[39] Tang, D., Zhu, Y. and Xie, Y. (2006) Investigation on Discoloration of Liqour by Solid Fermentation under Alkaline Conditions. Liquor-Making Science \& Technology, 7, 67-68.

[40] Zhang, R., Yin, C., Wang, G. and Gao, M. (2011) Effect of Alcoholicity on Changes of Absorbance of Heated Pure Grain Liquor under Alkaline Conditions. China Brewing, 30, 83-85. 